länger als 14 Tage fortgesetzt wurde. Nach einer Mittheilung von Kraut (Journ. f. Landwirthsch. 1858, Heft IX; Chem. Centralbl. 1858, 831) beobachtete Uelsmann aufallender Weise bei auf der Weide ernährten Kühen der Eldenaer Gutswirthschaft drei Wochen hindurch einen kohlensäurefreien und sauer reagirenden Harn. - Wir haben das vollständige Verschwinden der Kohlensäure und die begleitende saure Reaction des Harns bei Fütterung von Weizenstroh durch einen Controlversuch bestätigt und gefunden, dal's ein Zusatz zu der Strohration von 75 Grm. essigsaurem Kali die Kohlensäure und damit die alkalische Reaction des Harns wieder zum Vorschein brachte.

(Mai 1862.)

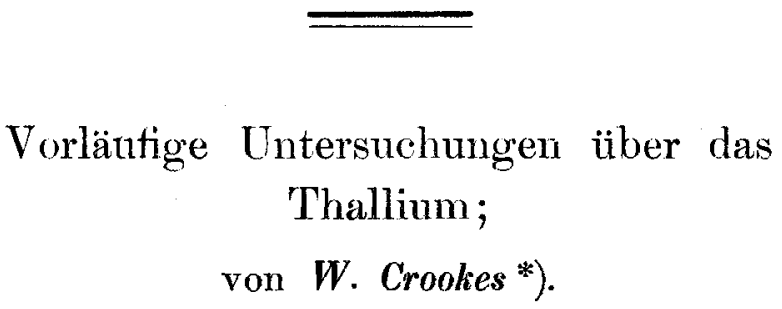

(Auszug ans einer vor der Royal Society zu London am 19. Juni 1862 gelesenen Abhandlung.)

Das Vorkommen einer grünen Linie in dem Spectrum einiger Selen-Rückstände, welche ich auf einen Tellurgehalt untersuchte, gab mir zuerst Veranlassung, die Anwesenheit eines neuen Metalls zu vermuthen. Im März 1861 **) kündigte ich in bestimmter Weise an, dafs die Substanz, welcher

*) Chemical News VI, 1 (v. 5. Juli 1862).

**) Philosophical Magazine [4] XXI, 301 und Chemical News III, 194 (v. 30. März 1861). 
die grüne Spectrallinie angehört, gewifs ein neues Element sei. Der Aufsatz, in welchem ich diefs that, enthielt eine hinreichende Anzahl Reactionen dieser Substanz, um mich in den Stand zu setzen, auf chemischem Wege so gut als auf optischem den Beweis zu führen, dafs ich mit einem, scharf bestimmte Eigenschaften besitzenden Elemente zu thun hatte. Ich setzte die Untersuchung fort und konnte in dem folgenden Mai *) weitere Nachricht über diese Substanz geben, für welche ich die Bezeichnung Thallium (mit dem Zeichen Tl) vorschlug. In diesem Anfsatz gab ich auch die Localitäten und die Beschreibung verschiedener Mineralien, in welchen ich das neue Element gefunden hatte, und beschrieb auch ein Verfahren, es daraus im reinen Zustande darzustellen. In Betracht, dafs ich die Entdeckung durch diese Aufsätze, welche in der Mehrzahl der chemischen Zeitschriften in Europa wiedergegeben wurden, genugsam angekündigt hatte, richtete ich meine Aufmerksamkeit auf die Auffindung einer Quelle von Thallium, welche mir die Darstellung dieses Körpers in gröfserem Mafsstabe erlaube; meine Versuche waren bis dahin auf die Bearbeitung einiger mineralogischer Handstücke beschränkt, für welche ich nur schwierig die Herkunft ermitteln konnte, und die ganze Menge Thallium, die ich bis dahin erhalten hatte, betrug nicht mehr als drei Grains.

Etwas später war Herr Thornthwaite so gefällig, mich mit einer beträchtlichen Quantität rohen Schwefels zu versehen, welcher aus spanischen kupferhaltigen Kiesen destillirt war. Ich fand hierin einen Thalliumgehalt von 1 bis 2 Grains auf das Pfund, und bis vor einigen Monaten habe ich mit dem neuen Element gearbeitet, das aus diesem Rohmaterial dargestellt war. Ich habe indessen in neuerer Zeit ein

*) Chemical News III, 303 (v. 18. Mai 1861). 
thalliumhaltiges Erz aufgefunden, das in England bearbeitet wird und aus welchem ich das neue Metall in gröfseren Quantitäten darzustellen hoffe.

Folgendes Verfahren zur Darstellung des neuen Elementes aus Schwefel oder Kiesen fand ich am Vortheilhaftesten :

Das Erz wird fein gepulvert und möglichst vollständig in starker Salzsäure unter zeitweisem Zusatz von Salpetersäure gelöst, bis sich Nichts mehr weiter auflöst; dann wird mit Wasser verdünnt und filtrirt. Es wird zur Verjagung der überschüssigen Salpetersäure eingedampft, wenn nöthig unter Zusatz von etwas Schwelelsäure und unter Beachtung, dafs die Lösung nicht zur Trockne kommen oder auch nur taigig werden darf. Es wird dann mit Wasser verdünnt und etwas erwärnt, damit man sicher sei, dals Alles Lösliche sich wirklich in Lösung befinde. Es wird filtrirt; bei Anwesenheit von Blei bleibt der gröfste Theil desselben in Form von unlöslichem schwefelsaurem Salz auf dem Filter. Das Filtrat wird stark verdünnt, und eine Lösung von kohlensaurem Natron zugesetzt, bis die Reaction eine deutlich alkalische ist; dann wird ein Ueberschufs von Cyankaliumlösung (welche frei von Schwefelkalium sein mufs) zugefügt. Es wird eine Zeit lang mäfsig erhitzt und dann filtrirt. Der Niederschlag enthält alles Blei und Wismuth, das etwa anwesend ist, in der Form von kohlensauren Salzen, während das Thallium in Lösung ist. Ein nun in die Flüssigkeit geleiteter Strom von Schwefelwasserstoffgas fällt alles Thallium aus, während Kupfer, Antimon, Zirn und Arsen gelöst bleiben. Wenn Cadmium und Quecksilber anwesend sind, so gehen sie mit dem Thallium in den Niederschlag. Das erstere Metall kann leicht mittelst warmer verdünnter Schwefelsäure ausgezogen werden, welche auf Schwefelthallium kaum lösend einwirkt, während dieses wiederum von dem Schwefelquecksilber durch Kochen in mälsig verdünnter Salpetersäure getrennt 
werden kann, in welchem das Schwefelquecksilber unlöslich ist. Diese beiden Metalle finden sich jedoch nur selten in den von mir untersuchten thalliumhaltigen Erzen. Die salpetersaure Lösung wird nun zur Trockne eingedampft, der Rückstand in heifser verdünnter Schwefelsäure gelöst und in diese Lösung ein Stück reinen metallischen Zinks gebracht; das Thallium wird sofort in Form eines tiefbraunen Pulvers ausgefällt, welches sich bald zu einem schweren schwarzen körnigen Niederschlag urnwandelt. In cohärenter Form läfst sich das Metall durch Schmelzen in Wasserstoffgas erhalten.

Das hier beschriebene Verfahren gilt für den Fall, dafs alle genannten Metalle anwesend sind. In den meisten Fällen ist es beträchtlicher Abkürzung fähig, da die Erze selten so complicirte Zusammensetzung besitzen. Wenn die Beschaffung von vollkommen reinem Zink für die Reduction des Metalls aus dem schwefelsauren Salz Schwierigkeiten unterliegt, so kann die Reduction auch in der Art bewirkt werden, dal's man, unter Anwendung von Platinpolen, einen schwachen electrischen Strom durch die Flüssigkeit gehen lälst; das Metall wird dann als Regulus oder schwammig ausgefällt, je nach der Stärke des Stroms. Versuche, das Metall aus dem Oxyd mittelst Wasserstoff zu reduciren, waren nicht sehr erfolgreich; der Gasstrom reifst das flüchtige Oxyd mit sich von dem erhitaten Theile der Röhre weg, bevor vollständige Reduction stattgefunden hat. Es ist jedoch, wie ich gegen das Ende dieser Versuche fand, wahrscheinlich, daf's bei Anwendung einer im Verhältnifs zu der Menge Substanz längeren Röhre, die in ihrer ganzen Länge stark erhitzt ist, sich auf diese Art doch gute Resultate erhalten lassen mögen; denn das Metall ist beträchtlich weniger flüchtig als das Oxyd.

In einigen Fällen, wenn nur sehr geringe Spuren von 
Thallium grofse Quantitäten anderer Metalle begleiten, kann es rathsam sein, alle die oben beschriebenen Operationen oder einige derselben zu wiederbolen, um dieses Element von fremden Metallen, die bei einmaliger Ausführung jener Operationen noch nicht vollständig beseitigt waren, rein zu erhalten.

Ich gehe nun über zu der Beschreibung des Thalliums und seiner chemischen Reactionen.

Das Thallium ist im reinen Zustand ein schweres Metall, welches bezüglich seiner physikalischen Eigenschaften eine bemerkenswerthe Aehnlichkeit mit dem Blei hat. Sein specifisches Gewicht ist jedoch höher, etwa $=12$. Die frisch geschabte Oberfläche zeigt beträchtlichen Metallglanz, ohne dafs die Farbe so bläulich ist wie bei dem Blei; eine solche Oberfläche verliert den Glanz rascher, als diefs bei dem Blei der Fall ist. Das Thallium ist sehr weich, läfst sich leicht mit einem Messer schneiden und nimmt von dem Nagel Eindrücke an; es läfst sich auch aushämmern und zu Draht ziehen, zeigt aber in dieser Form nicht grofse Festigkeit. Es färbt leicht auf Papier ab. Der Schmelzpunkt liegt unter der Rothglühhitze, und bei einiger Sorgfalt lassen sich verschiedene Stücke zu Einem Klumpen zusammenschmelzen. Doch findet bei dieser Operation im Allgemeinen Verlust statt, in Folge der raschen Oxydation des Metalls. Das Thallium selbst scheint unterhalb der Rothglühhitze nicht bemerklich flüchtig zu sein. Ich habe bis jetzt noch keine Versuche zum speciellen Zweck der Bestimmung des Atomgewichtes gemacht, welches indessen, nach zwei Ermittelungen des Schwefelgehaltes im Schwefelthallium, ein sehr hohes zu sein scheint; doch stimmen die hierbei erhaltenen Zahlenresultate nicht genug unter sich überein, um mich mehr hierüber aussprechen zu lassen, als dafs das Atomgewicht gröfser als $100 \mathrm{zu}$ sein scheint. Ich darf anführen, 
dafs ich das neue Element im reinen metallischen Zustand bereits im Januar dargestellt und mehreren Freunden gezeigt habe *), und dafs ich damals schon eine Beschreibung desselben veröffentlicht hätte, wenn nicht die oben besprochenen Umstände mich davon abgehalten hätten. Das Thallium löst sich in Salpetersäure, Salzsäure und Schwefelsäure ; die erstere Säure greift es mit grofser Energie und unter Entwickelung rother Dämpfe an.

Oxyde des Thalliums. - Das Thallium bildet zwei, wahrscheinlich selbst drei Oxyde. Eines derselben besitzt basische Eigenschaften, und dieses werde ich Thalliumoxyd nennen; ein anderes sauerstoffreicheres besitzt saure Eigenschaften und mag defshalb als Thalliumsäure bezeichnet werden. Wahrscheinlich existirt noch eine dritte Oxydationsstufe, ein Suboxyd, welches die ersten Portionen der bei Einwirkung von Zink auf Thalliumlösungen sich bildenden Ausscheidung ausmacht; bei dieser Einwirkung färbt sich zuerst die Lösung dunkel und ein tief braunes Pulver scheidet sich aus, tas bei längerer Berührung mit Zink zu einem dichten schwarzen Niederschlag wird.

Wenn man die salpetersaure Lösung im Wasserbade behutsam eindampft, ohne sie jedoch zur Trockne zu bringen, so entsteht beim Abkühlen eine Masse zerfliefslicher Krystalle, welche auf Zusatz von Wasser unter Ausscheidung eines weifsen oder blafsgelben Niederschlages zersetzt werden, der ein basisches salpetersaures Salz zu sein scheint; die über diesem Niederschlag stehende saure Lösung enthält salpetersaures Thallium. Wird die Flüssigkeit ganz zur Trockne eingedampft und der Rückstand während einiger Zeit bei $100^{\prime \prime} \mathrm{C}$. erbalten, so geht die Salpetersäure weg und es bleibt Thalliumsäure zurück.

*) Vgl. Chemical News V, 349 น. $\$ 50$ 
Thalliumsäure. - Diese Säure ist löslich in Wasser und kann aus der wässerigen Lösung in krystallinischer Form erhalten werden. Sie bildet dann Krystalle, welche luftbeständig sind und auf Reagenspapier sauer reagiren. Die thalliumsauren Salze der Alkalien sind gleichfalls löslich in Wasser; sie lassen sich darstellen durch Lösung der Säure in dem Alkali oder durch Schmelzen des Thalliums oder seines Oxyds mit einem Gemenge von kohlensaurem und salpetersaurem Alkali. Das von mir zuerst veröffentlichte Verfahren zur Ausziehung des Thalliums war darauf gegründet, dafs sich in dieser Weise ein in Wasser lösliches thalliumsaures Alkalisalz bilden läfst. Die Thalliumsäure entsteht auch in wässeriger Lösung bei Zusatz von übermangansaurem Kali zu einem löslichen Thalliumoxydsalz.

Chlorthallium. -- Bei dem Ueberleiten eines Stromes von trockenem Chlorgas über gefälltes Thallium bei mäfsiger Hitze verbinden sich beide Substanzen unter Bildung eines flüchtigen Chlorides, welches sich in dem kälteren Theile der Röhre in Form eines blafsgelben krystallinischen Pulvers absetzt, das theilweise zu einem krystallinischen Klumpen zusammenschmilzt. In Wasser löst sich dieses Product nur theilweise, unter Hinterlassung eines weifsen unlöslichen Rückstandes. Auf Zusatz von verdünnter Salzsäure zu der trüben Lösung wird diese sofort klar. Bei dem Abdampfen dieser Lösung auf dem Wasserbad werden weifse Krystalle ausgeschieden. Wenn man die salpetersaure Lösung von Thallium oder Schwefelthallium mit überschüssiger Salzsäure eindampft, dann noch mehr Salzsäure zusetzi und das Eindampfen wiederholt bis der Rückstand Syrupconsistenz angenommen hat, so erhält man ein Product, das anscheinend durch Wasser, unter Bildung eines weilsen Niederschlags, zersetzt wird. Letzterer ist Thalliumchlorid; es ist in Wasser unlöslich oder 
fast unlöslich, in verdünnter Salzsäure oder Salpetersäure leicht löslich.

Schwefelthallium. - Bei dem Durchleiten von Schwefelwasserstoff durch die saure Lösung von Chlorthallium findet eine theilweise Ausscheidung eines röthlichbraunen Pulvers statt. Dieses scheint eine Verbindung von Chlorthallium mit Schwefelthallium zu sein, und das Metall wird aus seiner Lösung auf diese Art niemals vollständig ausgefällt. Das beste Verfahren, das Schwefelthallium darzustellen, besteht darin, es aus einer alkalischen Lösung mittelst Schwefelammonium auszufällen. Wenn nicht eine sehr grofse Menge Thallium in der Lösung enthalten ist, zeigt sich zunächst nur ein Dunkelfärben der Flüssigkeit; die eintretende braune Färbung wird rasch intensiver, namentlich bei mäfsigem Erwärmen, bis sich das Schwefelthallium in Form eines tief braunen schweren Niederschlags ausscheidet, der grofse Neigung zeigt sich am Boden des Gefäfses zu Klumpen zu vereinigen; die Bildung dieser Schwefelverbindung ist sehr characteristisch für das Thallium. Schwefelthallium ist unlöslich in einem Ueberschufs von Schwefelammonium, in Ammoniak und in Cyankalium. Dafs das Thallium aus Lösungen, welche einen Ueberschufs von Cyankalium enthalten, sich als Schwefelthallium vollständig ausfällen läfst, giebt ein sehr brauchbares Hülfsmittel ab, das Thallium von verschiedenen Metallen zu scheiden, mit denen es häufig zusammen vorkommt. Das Schwefelthallium löst sich nur schwierig in Salzsäure oder Schwefelsäure, aber leicht in Salpetersäure. Trocken ist es ein tief braunes, fast schwarzes Pulver, welches beim Erhitzen schmilzt und sich verflüchtigt. Im reinen Zustand ist es weder so schmelzbar noch so flüchtig wie Schwefel; aber wenn es mit einem Ueberschusse des letzteren Elementes gemischt ist, hat es grofse Schwierigkeiten es durch Sublimation von diesem zu scheiden. 
Rohlensaures Thallium scheidet sich bei Zusatz eines kohlensauren Alkalisalzes zu der sauren Lösung des Chlorids aus. Es ist mäfsig löslich in einem Ueberschufs von kohlensaurem Ammoniak, leicht löslich in Cyankalium. Dieses ist eine sehr bestimmte Reaction, welche dasi Thallium mit Schärfe von Blei und Wismuth zu scheiden gestattet.

Schwefelsaures Thallium. - Wird eine salzsaure oder salpetersaure Lösung von Thallium mit Schwefelsäure eingedampft, so entweicht die flüchtigere Säure und das schwefelsaure Salz bleibt zurück. Es ist löslich in Wasser.

Jodthallium wird bei vorsichtigem Zusatz von Jodkalium zu einer Thalliumlösung als ein gelblich - rothes Pulver ausgefälit. Es ist leicht löslich in einem Ueberschufs von Jodkalium, unter Bildung einer farblosen Flüssigkeit.

Phosphorsaures Thallium bildet einen weifsen flockigen Niederschlag, welcher in Mineralsäuren löslich, aber in Essigsäure nur wenig löslich ist.

Ferrocyanthallium ist weifs und unlöslich in Wasser.

Cyanthallium wird bei vorsichtigem Zusatz von Cyankalium zu einer Thalliumlösung als ein weifses oder hellbraunes Pulver niedergeschlagen. Es ist leicht löslich in einem Ueberschufs des Fällungsmittels.

Chromsaures Thallium ist ein blafsgelber Niederschlag; es löst sich in Säuren und wird bei Neutralisation derselben mit Ammoniak ausgefällt.

Keine Niederschläge bilden sich bei dem Versetzen einer Thalliumlösung mit Zinnchlorür, Oxalsäure, Pikrinsäure, schwefliger Säure oder schwefelsaurem Eisenoxydul.

Die meisten dieser Reactionen sind durch meine Freunde E. 0. Brown und J. Spiller bestätigt worden, und ich spreche Denselben gern bei dieser Gelegenheit meinen Dank für die schätzbare Hülfe aus, welche sie mir leisteten. Die angeführten Reactionen sind hinreichend, um den chemischen 
Beweis zu führen, dafs der hier in Retrachtung stehende Körper ein neues Element ist. Sein Verhalten im Spectralapparat giebt wohl die endgültigste Entscheidung bezüglich dieses Punktes ab. Wird eine kleine Menge der Schwefeloder der Chlorverbindung, oder in der That irgend einer Verbindung des Thalliums in die Flamme des Spectroscops eingeführt, so läfst sie sofort eine einzelne grüne Linie erscheinen, welche vollkommen scharf und bestimmt auf schwarzem Grunde, in aufserordentlicher Reinheit und Intensität, fast so glänzend wie die Natriumlinie auftritt. Doch ist diese Erscheinung nicht länger dauernd. In Folge der grofsen Flüchtigkeit zeigt eine auf einmal in die Flamme gebrachte Portion die grüne Linie nur als eine glänzende, rasch vorübergehende Lichterscheinung, die nur einen Bruchtheil von einer Secunde dauert; wird aber die Thalliumverbindung allmälig in die Flamme gebracht, so zeigt sich die Linie während viel längerer Zeit. Auch wenn ein Stückchen metallisches Thallium in einem Platindraht-0ehr in die Flamme gebracht wird, wo das Platin und das Thallium zusammenschmelzen, giebt die Legirung die grüne Linie ziemlich dauernd, wenn auch natürlich schwächer.

Bei dem Arbeiten in kleinerem Mafsstab ist es nicht leicht, diese Verbindungen frei von Natron zu erhalten; ist diefs jedoch gelungen und wird eine mäfsige Menge Substanz auf einem Platindraht-Oehr in eine Flamme gehalten, so ist die grüne Färbung derselben höchst glänzend und dieselbe bewirkt ganz aufserordentliche Effecte in dem Aussehen der ungebenden Gegenstände. Wenn Thallium in gröfseren Mengen gewonnen werden könnte, würde dieses Mittel zur Hervorbringung eines intensiven und homogenen grünen Lichtes gewifs nützlicher Anwendungen fähig sein.

Die grüne Linie im Thalliumspectrum scheint von keiner anderen Linie oder Streifen in anderen Theilen des Spectrums 
begleitet zu sein. Eine Flamme von genügend hoher Temperatur, dafs sie die orangefarbene Linie des Lithiums sichtbar macht, fügt der Einen Thalliumlinie keine andere hinzu; und bei Anwendung eines so stark vergröfsernden Fernrohrs, dafs die beiden Natriumlinien durch einen beträchtlichen Zwischenraum von einander getrennt sind, zeigt sich die Thalliumlinie noch als eine einfache. Ich glaube defshalb gerechtfertigt zu sein, wenn ich ausspreche, dafs unter allen bekannten Elementen das Thallium das einfachste Spectrum giebt. Theoretische Untersuchungen über die Ursache der Linien im Spectrum und über die Beziehungen derselben zu anderen constanten Eigenschaften eines Elementes dürften nun erleichtert sein, da wir jetzt ein Element kennen, welches nur Lichtschwingungen von Einem Grad der Brechbarkeit entstehen läfst. Die bemerkenswerthe Einfachheit des Thalliumspectrums steht in starkem Gegensatz zu den verwickelten Spectren, welche durch Quecksilber, Wismuth und Blei - die Metalle, mit welchen das Thallium in chemischer Beziehung am meisten Aehnlichkeit hat - hervorgebracht werden.

Die Lage der grünen Linie coüncidirt nicht mit der irgend einer schärferen Linie im Sonnenspectrum. Nach Kirchh off's Theorie wäre somit anzunehmen, dafs Thallium nicht, oder wenigstens nicht in gröfserer Menge, in der Sonne anwesend sei. Unter der stärksten Vergröfserung meines Apparates scheint die grüne Thalliumlinie bezüglich der Brechbarkeit vollkommen identisch zu sein mit einer scharfen, gut bestimmten Linie im Baryumspectrum, welche Bunsen und Kirchhoff als $\mathrm{Ba}_{\text {s }}$ bezeichnet haben. Mangel an Material hat mich bisher verhindert, die Distanzen zwischen der Thalliumlinie und den hauptsächlichsten Linien des Sonnenspectrums genau zu messen.

Diese grüne Linie ist ein äufserst empfindliches Erkennungsmittel für die Anwesenheil des Thalliums, und es läfst sich 
mittelst desselben nachweisen, dafs das Thallium ein ziemlich weit verbreitetes Element ist. Verschiedene Proben, rohen Schwefels, namentlich dunkel-aussehenden, enthielten es. In den meisten Fällen ist es nur nöthig, ein so grofses Stück Schwefel (doch nicht gröfser als eine Erbse), als das Platindraht-Oehr halten kann, anzuzünden, und, wenn der Schwefel fast ganz weggebrannt ist, ihn auszublasen und das noch Rückständige in die Flamme des Spectroscops zu bringen, wo das Thallium seine Anwesenheit dadurch anzeigt, dafs es in dem Gesichtsfeld die helle grüne Linie für einen Augenblick aufblitzen läfst. Wenn auch der gröfsere Theil des Thalliums sich in dem Rückstand noch findel, welcher bei dẹm Verbrennen des Ueberschusses des Schwefels in dieser Weise bleibt, so verflüchtigt sich doch auch etwas davon; und es ist somit, wenn eine Probe bei diesem Verfahren die Thalliumreaction nicht giebt, rathsam, einen möglichst grofsen Theil des Schwefels mittelst Schwefelkohlenstoff auszuziehen und den Rückstand spectralanalytisch zu prüfen.

Thallium ist in sehr vielen Erzen enthalten. Bei der Untersuchung einer sehr grofsen Sammlung kupferhaltiger Kiese aus verschiedenen Theilen der Erde fand ich es in mehr als Einem Achtel derselben. Es ist nicht auf eine besondere Localität beschränkt, und sein Vorkommen scheint auch nicht zu der Anwesenseit oder Abwesenheit von Arsen in dem Mineral in einer Beziehung zu stehen. Ich habe es jedoch nur selten in solchen Kiesen gefunden, welche frei von Kupfer waren. Es ist meistens nur nöthig, ein kleines Stück des Minerals zu pulvern und Etwas davon auf einem befeuchteten Platindraht in der Flamme zu erhitzen, damit die grüne Linie deutlich sichtbar auftrete.

Wird ein thalliumhaltiger Kies fein gepulvert und dann in einer Glasröhre unter möglichstem Abschlufs der Luft zum 
Rothglühen erhitzt, so sublimirt Schwefelthallium zugleich mit etwas freiem Schwefel und kann in geeigneter Weise verdichtet werden. Dieses Sublimat giebt die Thalliumlinie in sehr glänzender Weise.

\title{
Ueber ein neues Metall, das Thallium;
}

\author{
von A. Lamy*).
}

Als ich vor einigen Monaten mittelst $\mathrm{Kirch}$ off und Bunsen's spectralanalytischen Apparats eine Probe Selen untersuchte, welches mein Schwager, Herr F. Kuhlmann, aus dem Schlamme der Bleikammern, in welchen man durch Verbrennung von Schwefelkiesen Schwefelsäure bereitet, dargestellt hatte, bemerkte ich eine scharfe grüne Linie, welche mir bei keiner der von mir untersuchten zahlreichen unorganischen Substanzen von einfacher oder zusammengesetzter Natur vorgekommen war. Es war mir damals unbekannt, dafs ein englischer Chemiker, Herr W. Crookes, nicht allein dieselbe grüne Linie unter fast ganz ähnlichen Umständen entdeckt, sondern dafs er auch das neue Element, für welches diese grüne Linie im Spectrum characteristisch ist, bereits als Thallium bezeichnet hatte, von dem griechischen Worte $9 \alpha \lambda \lambda 0_{s}^{\prime}$ oder dem lateinischen Worte thallus, welches man oft gebraucht, um die Färbung einer jungen kräftigen Vegetation zu bezeichnen. Mit grofsem Scharfsinn hatte Herr Crookes bereits einige der Reactionen dieses Elementes

*) Compt. rend. LIV, 1255 (Sitzung der Pariser Academie Fom 23. Juni 1862). 